ORIGINAL ARTICLE

\title{
Insect sting anaphylaxis; prospective evaluation of treatment with intravenous adrenaline and volume resuscitation
}

\author{
S G A Brown, K E Blackman, V Stenlake, R J Heddle
}

Emerg Med J 2004;21:149-154. doi: 10.1136/emj.2003.009449

See end of article for authors' affiliations .....................

Correspondence to: Dr S G A Brown, Department of Emergency Medicine, Fremantle Hospital, Alma Street, Fremantle, Western Australia; simon.brown@ health.wa.gov.au

Accepted for publication 12 September 2003

\begin{abstract}
Objectives: To assess a protocol for treatment of sting anaphylaxis.
Design: Prospective assessment of treatment with oxygen, intravenous infusion of adrenaline (epinephrine), and volume resuscitation with normal saline.

Setting: Sub-study of a venom immunotherapy trial.

Participants: 21 otherwise healthy adults with systemic allergic reactions to diagnostic sting challenge.

Main outcome measures: Response to treatment, total adrenaline dose and infusion duration, recurrence of symptoms after stopping the infusion, and additional volume resuscitation.

Results: 19 participants required intervention according to the protocol. All received adrenaline, and five received volume resuscitation. In nine cases, physical signs of anaphylaxis recurred after initial attempts at stopping adrenaline but resolved after recommencing the infusion. The median total dose and infusion duration were $590 \mu \mathrm{g}$ and 115 minutes respectively, but were significantly higher for eight patients who had hypotensive reactions $(762 \mu \mathrm{g}$ and 169 minutes respectively). Hypotension was always accompanied by a relative bradycardia, which was severe and treated with atropine in two patients. Widespread T wave inversion occurred, before starting treatment with adrenaline, in one person with an otherwise mild reaction. All patients fully recovered and were fit for same day discharge, apart from the person with ECG changes who was observed overnight and discharged the following day.

Conclusions: Carefully titrated intravenous adrenaline combined with volume resuscitation is an effective strategy for treating sting anaphylaxis, however severe bradycardia may benefit from additional treatment with atropine. Cardiac effects of anaphylaxis, perhaps including neurocardiogenic mechanisms, may be an important factor in some lethal reactions.
\end{abstract}

$\mathrm{H}$ uman data on the efficacy and safety of pharmacological treatments for anaphylaxis are limited. Management guidelines, which emphasise a central role for adrenaline (epinephrine), ${ }^{1-3}$ are based largely on expert opinion. Reactions can spontaneously resolve with endogenous compensatory responses, ${ }^{4}$ but failure to use adrenaline has been considered a major factor contributing to lethal outcomes. ${ }^{5}$ Retrospective analysis of 205 episodes of anaphylactic shock occurring under anaesthesia indicates that adrenaline and volume resuscitation are the cornerstones of successful resuscitation. ${ }^{7}$ However, clinical observations of severe anaphylaxis in humans, ${ }^{8}$ as well as canine experiments, ${ }^{9}$ suggest that a single dose of adrenaline may produce only transient improvement. There have been recurrent debates on the indications for, and optimal route of administration of adrenaline. Commentaries emphasise the need to adapt to prevailing clinical conditions; clinical urgency, degree of circulatory compromise, availability of vascular access, and the level of care available. ${ }^{10-12}$

While performing a trial of venom immunotherapy we undertook this sub-study that aimed to assess a sting anaphylaxis treatment protocol consisting of supplemental oxygen, a carefully titrated intravenous adrenaline infusion, and volume resuscitation.

\section{METHODS}

Our randomised double blind, placebo controlled crossover trial of Myrmecia pilosula venom immunotherapy has been described in detail elsewhere. ${ }^{13}$ Briefly, 68 participants with a history of systemic reactions to $M$ pilosula were randomly allocated to either placebo or immunotherapy with a venom extract. Eligibility criteria were; (1) age between 17 and 65 years; (2) a history of grade II-IV hypersensitivity to a clearly identified or presumed $M$ pilosula sting (boxl); (3) a positive intradermal venom skin test; (4) no history of hypertension, heart disease, poorly controlled lung disease, ACE inhibitor or $\beta$ blocker therapy, and; (5) willingness to undergo a sting challenge with the risk of severe, potentially life threatening anaphylaxis. After immunotherapy, a diagnostic sting challenge was performed in a resuscitation room under the supervision of an emergency physician to determine the efficacy of immunotherapy in preventing reactions.

The inclusion of a placebo group was considered essential because without demonstrating an ability to precipitate anaphylaxis, we could not be certain that the sting challenge would be an adequate test of immunotherapy. Failure to rigorously determine efficacy of immunotherapy could lead to a false sense of security in patients, a contributing factor in at least one recent death in our region. ${ }^{15}$ Furthermore, the natural history of sting allergy is unpredictable, and many people (as many as $75 \%$ in the case of wasp sting allergy) lose their sensitivity over time. ${ }^{16}{ }^{17}$ Therefore, a concurrent control group was required for statistical analysis. On the basis of previous large sting challenge studies we considered this to be a comparatively safe procedure if strict exclusion criteria were applied..$^{16}{ }^{17}$ Finally, the potential long term benefit to participants of developing an effective immunotherapy outweighed the small additional risk imposed by the trial. The relevant ethics committees approved the trial and fully informed written consent was obtained from each participant.

Before the sting challenge we performed baseline observations, including 12 lead ECG and spirometry. After inserting intravenous cannulas and preparing infusions we pushed a single ant against the ventral forearm and allowed it to sting 
Box 1 Grading of systemic allergic reactions after U R Müller ${ }^{14}$

I. Generalised urticaria* or erythema, itching, malaise, or anxiety

II. Angioedema* or two or more of: chest or throat tightness, nausea, vomiting, diarrhoea, abdominal pain, dizziness

III. Dyspnoea, wheezing or stridor, or two or more of: dysphagia, dysarthria, hoarseness, weakness, confusion, feeling of impending disaster

IV. Hypotension, collapse, loss of consciousness, incontinence of urine or faeces, or cyanosis $\dagger$

*Periorbital oedema was judged a manifestation of urticaria, not angioedema.

†During diagnostic sting challenges hypotension, defined by a systolic blood pressure $<90 \mathrm{~mm} \mathrm{Hg}$, was required to define a grade IV reaction.

for 60 seconds. During the sting challenge we monitored ECG, pulse oximetry, non-invasive blood pressure (NIBP), and ST segments. ST segment monitoring was with either a 5 lead system (alarms set at $0.5 \mathrm{mV}$ ) or 15-30 minutely 12 lead ECGs. Spirometry was repeated at 15 and 60 minutes after the sting, and at additional time points to quantify any respiratory symptoms.

Treatment using our protocol (box 2) focused on oxygen, intravenous adrenaline infusion, and volume resuscitation. The indications for starting treatment were designed to enable an accurate grading of reaction severity for the purposes of the venom immunotherapy trial. These were; (1) pulse oximetry saturation under $92 \%$ breathing room air; or (2) peak expiratory flow rate less than $60 \%$ of baseline; or (3) systolic blood pressure under $90 \mathrm{~mm} \mathrm{Hg}$; or (4) any evidence of myocardial ischaemia; or (5) stable reaction severity for 30 minutes, and; or (6) at the request of the trial participant. Reactions were graded according to box 1 . Antihistamines and corticosteroids were not part of routine management because their usefulness for the management of the hyperacute phase of anaphylaxis was unknown and because theoretical considerations indicated they were unlikely to be beneficial. Discharge home was permitted after a symptom free interval of at least two hours after withdrawal of the adrenaline infusion (longer for severe or complicated reactions), or one hour after sting challenge if no reaction occurred.

For each reaction we recorded the interval from sting to onset of first symptoms, individual reaction features and events during treatment, whether symptoms recurred after a first attempt at ceasing the infusion, total dose and duration of the adrenaline infusion, and other treatments administered. We also calculated the differences between baseline physiological values and the highest heart rate, lowest systolic and mean blood pressures, lowest pulse oximetry oxygen saturation, lowest peak expiratory flow, and lowest forced expiratory volume/forced vital capacity after sting challenge. Relations between clinical data elements and the total dose and duration of the adrenaline infusion were assessed with Analyse-it for Microsoft Excel (1.61 version, Analyse-it Software, Leeds, UK), using the Mann-Whitney U test and Spearman rank correlation for continuous variables and the $\chi^{2}$ test for categorical variables.

The following analysis is limited to the placebo group, as no reaction in the venom immunotherapy group required treatment with adrenaline.
Box 2 Treatment guidelines

1. Oxygen

- High flow oxygen $(15 \mathrm{I} / \mathrm{min})$ by facemask if $\mathrm{SpO}_{2}<92$ or $\mathrm{SBP}<90 \mathrm{~mm} \mathrm{Hg}$

\section{Adrenaline infusion}

- $1 \mathrm{mg}$ in $100 \mathrm{ml}(1: 100000,10 \mu \mathrm{g} / \mathrm{ml})$ intravenously by infusion pump

- Start at $30-100 \mathrm{ml} / \mathrm{h}(5-15 \mu \mathrm{g} / \mathrm{min})$ according to reaction severity

- Titrate up or down according to response and side effects, aiming for lowest effective infusion rate. Tachycardia, tremor, and pallor in the setting of a normal or raised blood pressure are signs of adrenaline toxicity; consider a reduction in infusion rate

- Stop infusion 30 minutes after resolution of all symptoms and signs

- Continue observation for at least two hours after ceasing infusion (longer for severe or complicated reactions); discharge only if remains symptom free

\section{Normal saline rapid infusion}

- $1000 \mathrm{ml}$ (pressurised) infused over 1-3 minutes and repeat as necessary

- Give if hypotension is severe or does not respond promptly to adrenaline

\section{Hypotension resistant to above measures*}

- Consider bolus adrenaline, glucagon $15-10 \mathrm{mg}$ IV bolus followed by infusion) and noradrenaline infusion with invasive blood pressure monitoring and central venous access.

*Planned contingencies, but not used during trial.

\section{RESULTS}

There were 21 systemic reactions in the placebo group. The time interval between sting and symptom onset ranged from 2-27 (median 8) minutes. The most common first symptoms were generalised itch and abnormal perioral sensations (tingling lips or tongue, or abnormal taste) in 10 and 7 people respectively. Erythema (with/without urticaria) was the initial physical sign in all cases apart from case 3 (see below).

There were seven grade I, three grade II, three grade III, and eight grade IV reactions. Skin features, although frequently subtle, were identified in all (generalised erythema $100 \%$, itch $82 \%$, urticaria $68 \%$ ). Angioedema occurred in seven (33\%), colicky abdominal pain (including severe "period-like" pains in one person) occurred in four (19\%), and respiratory features (dyspnoea, or wheeze) occurred in seven $(33 \%)$. One grade III and three grade IV reactions were accompanied by pulse oximetry saturations of $92 \%$ or less. Lowest measured systolic and mean pressures in grade IV reactions ranged from 0-88 (median 71) $\mathrm{mm} \mathrm{Hg}$ and $0-55$ (median 45) $\mathrm{mm} \mathrm{Hg}$ respectively. Hypotensive reactions were characterised by an initial fall in diastolic blood pressure, indicating systemic vasodilatation, and all were accompanied by an initial tachycardia followed by relative bradycardia with a heart rate drop of 15-65 (median 32 ) beats per minute accompanying the onset of hypotension. ST segment abnormalities occurred in two reactions (cases 3 and 4, see below). 
Adrenaline infusions were given for 19 reactions, including all those of grade II-IV severity. The two remaining urticarial reactions resolved without treatment. Five of the eight people with hypotension were also given a one litre saline bolus during the first few minutes of resuscitation. All responded rapidly to treatment, with symptomatic improvement and systolic blood pressure rising above $90 \mathrm{~mm} \mathrm{Hg}$ within five minutes except one who continued to deteriorate and was given a further two litres of saline (case 2, see below). Two below). There were no appreciable adverse reactions attributable to adrenaline. The median total dose of adrenaline was $590 \mu \mathrm{g}$ (range 190-1310 $\mu \mathrm{g}$ ) and median total infusion duration was 115 minutes (range 52-292 minutes). Total adrenaline doses and infusion durations are plotted against reaction grade in figure 1. Hypotensive reactions received significantly more adrenaline (median $762 \mu \mathrm{g} v 520 \mu \mathrm{g}$, $\mathrm{p}=0.02$ ) and longer infusions (median $169 \vee 92$ minutes, $\mathrm{p}=0.03$ ). Adrenaline dose and infusion duration did not correlate significantly with any other clinical parameter. In nine patients, seven of whom had grade III-IV reactions, the first attempt at ceasing the infusion was followed by a reaction recurrence and the infusion recommenced. This was after a median initial infusion time of 67 minutes in the group with symptom recurrence, compared with 79 minutes in those without recurrence, a non-significant difference. Corticosteroids and antihistamines were prescribed for three people; one with mild urticaria that kept recurring when the adrenaline was stopped, and two with very large local reactions at the sting site the following day.

Case 1 (fig 2A) illustrates a typical hypotensive reaction. A mild increase in heart rate follows the initial sting and then settles. The reaction begins with tachycardia and a fall in diastolic and mean blood pressures, indicating peripheral vasodilatation. Systolic hypotension and a slowing of the heart rate follow this.

Case 2 (fig 2B) was characterised by sudden visual loss and throbbing severe headache followed by hypotension. Despite rapid infusion of two litres saline over five minutes and adrenaline infused at $30 \mu \mathrm{g} / \mathrm{min}$, progressive bradycardia required treatment with atropine $600 \mu \mathrm{g}$ intravenously. At the same time, extravasation around the intravenous cannula was noted, where infusions had inadvertently been set on the same side that minutely blood pressure estimations were being performed. After swapping infusions to the back up cannula there was gradual improvement over the following 5-10 minutes. Notably, previous reactions had been characterised by visual loss and breathlessness without any other symptoms suggesting hypotension. These reactions responded promptly to intramuscular adrenaline. were given atropine for bradycardia (cases 2 and 3, see

Case 3 (fig 2C) began with a sensation of a "lump in the throat", followed within three minutes by unconsciousness, agonal respirations, and absent pulses. Atropine, adrenaline, and saline infusions were effective. Generalised erythema developed shortly after. One hour later an attempt was made to cease the adrenaline infusion but florid erythema developed accompanied by a fall in blood pressure. Inferior $\mathrm{T}$ wave inversion was noted on her ECG during the reaction; this resolved without any creatine kinase or troponin rises, and an outpatient exercise sestamibi scan was unremarkable.

Case 4 was a grade II reaction characterised by urticaria, angioedema, and chest tightness. There was no fall in either systolic or diastolic blood pressure, and heart rate did not rise above $110 \mathrm{bpm}$. ECG developed marked T wave inversion and mild ST depression (fig 3) prompting treatment with adrenaline. Although the ST depression improved with treatment, $\mathrm{T}$ wave inversion took several weeks resolve. Serial creatine and troponin were normal as was an outpatient exercise sestamibi scan.

\section{DISCUSSION}

We observed a consistent and rapid clinical improvement after starting treatment according to our protocol. The efficacy of adrenaline in particular was evident in the recurrence of reaction features on stopping the infusion, resolving rapidly again with its re-institution in nine cases. The fluid volumes we gave were less than reported from reactions occurring during anaesthesia, ${ }^{7}$ which may be a reflection of differences in antigen load and reaction severity, or blunted responsiveness attributable to anaesthetic agents. We did not identify any major adverse reactions to treatment, although in one case inadvertent infusion on the same side as frequent non-invasive blood pressure measurements probably delayed response to treatment and caused a degree of drug and fluid extravasation.

Significantly more adrenaline was used to treat hypotensive than non-hypotensive reactions. Relative bradycardia always accompanied hypotension and in two cases was treated with intravenous atropine; one appeared to be progressing towards cardiac arrest and the other had no detectable pulses. We observed one case where the myocardium was clearly involved in the anaphylactic process in the absence of circulatory instability, although it was impossible to determine whether this was secondary to coronary vasospasm or an effect of anaphylactic mediators on the myocardium. One reaction also suggested the nervous system as a target organ for anaphylaxis, with visual loss occurring before cardiovascular compromise.

The strengths of this study are that reaction features and response to treatment were monitored in a controlled
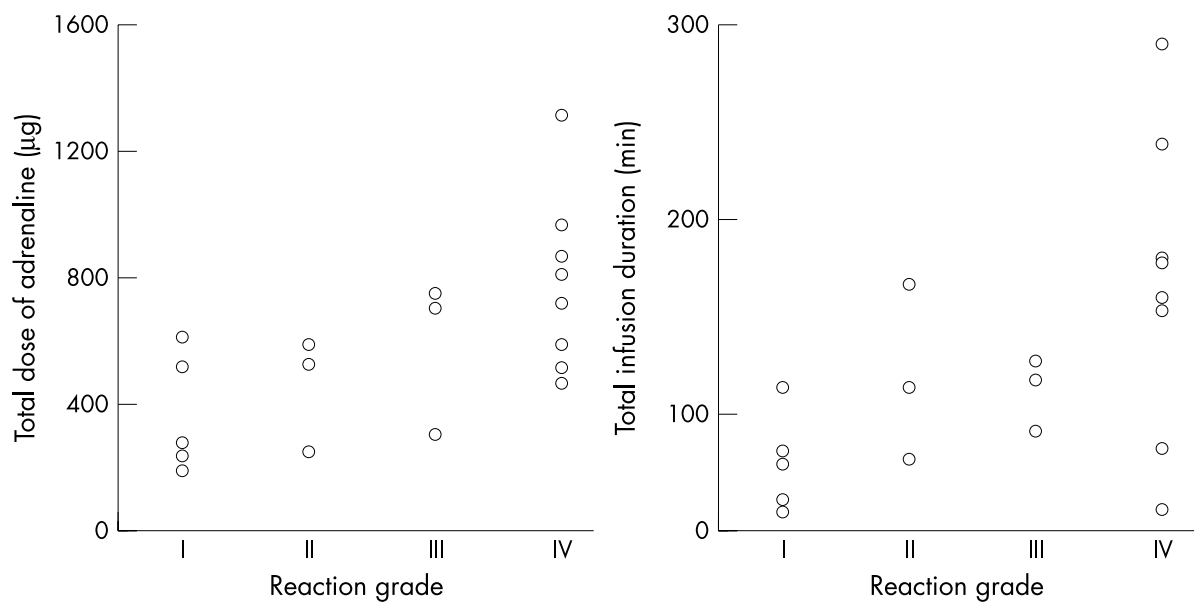

Figure 1 Total dose of adrenaline and total infusion duration by reaction grade. 
A

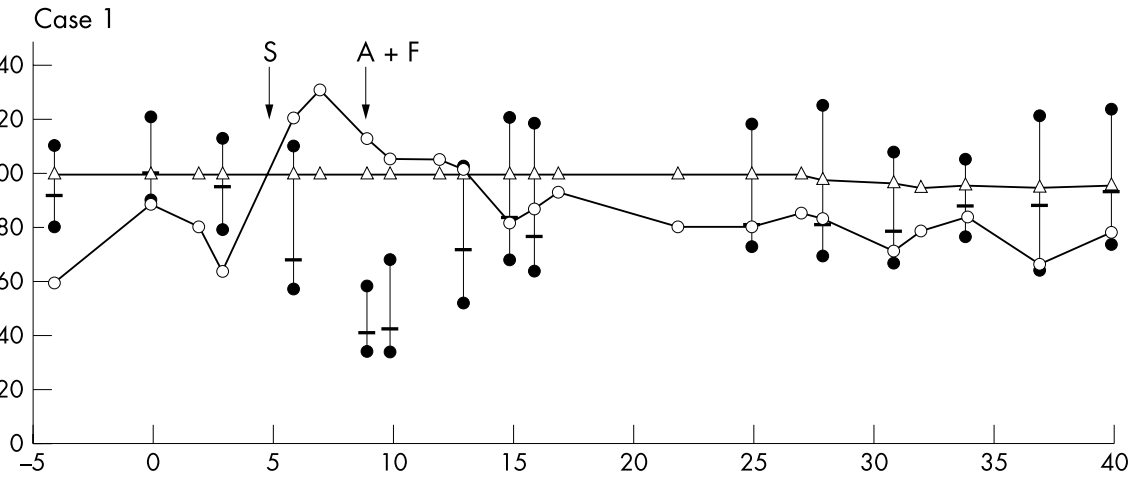

Figure 2 Observations and treatment for cases 1-3.

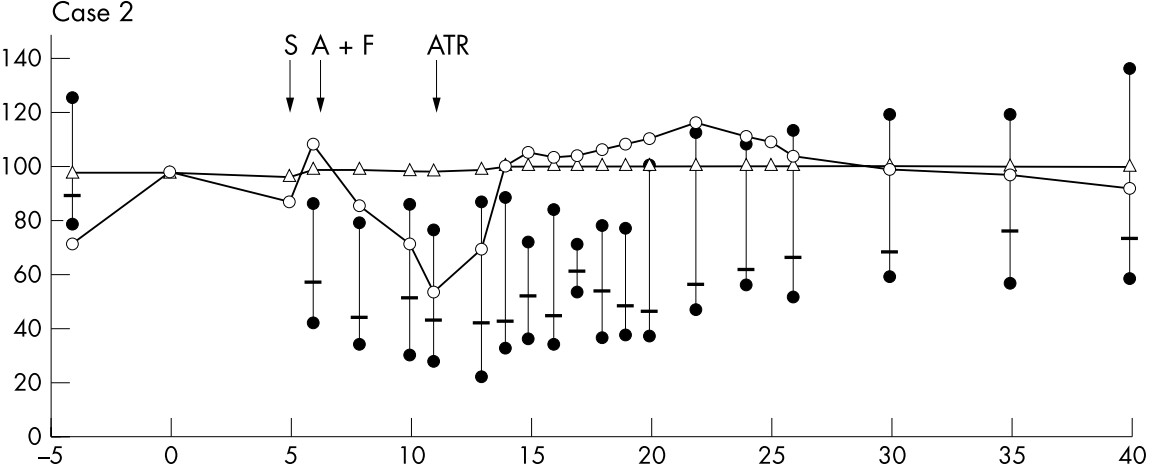

C

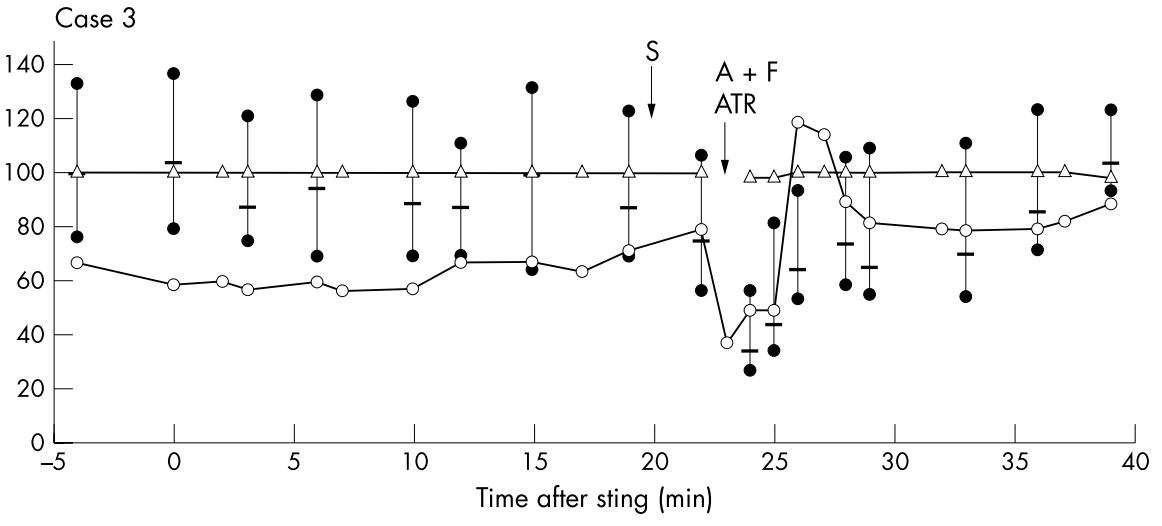

\begin{tabular}{|llll|}
$\longrightarrow \longrightarrow$ Heart rate & $\bullet$ BP-sys & $\mathrm{S}$ & First symptoms \\
$\longrightarrow-\mathrm{SpO}_{2}$ & BP-mean & $\mathrm{A}+\mathrm{F}$ & Adrenaline and fluid infusions started \\
& - BP-dia & ATR & Atropine $600 \mu \mathrm{g} \mathrm{IV}$ \\
\hline
\end{tabular}

environment according to a consistent protocol, a situation rarely possible when studying human anaphylaxis because of its infrequent and emergent nature. Weaknesses include the exclusion of patients with comorbidities and absence of a control (untreated) group, however these limitations were essential for patient safety. Also, the small number of cases prevented a confident assessment of how the various clinical parameters might predict the amount of adrenaline required.

The apparent success of our treatment protocol using adrenaline and volume resuscitation is consistent with findings by Fisher, who observed rapid improvement with adrenaline as well as evidence of fluid extravasation of up to $35 \%$ of circulating blood volume within 10 minutes of reaction onset. ${ }^{7}$ The rapid response of patients to our treatment protocol contrasts with a report of 17 patients with anaphylactic shock deliberately induced by insect sting and treated only with fluids and antihistamines, where "all but two recovered within 4 hours". ${ }^{4}$
Protracted anaphylaxis requiring large doses of adrenaline and noradrenaline after deliberate sting challenge has been reported. ${ }^{8}$ Health based exclusion criteria do not appear to have been applied in that study, where a large antigen load was also administered by graded subcutaneous injection before the sting-possibly contributing to the severity of the subsequent reactions. However, adrenaline resistant anaphylaxis has been recognised as an important albeit rare phenomenon probably associated with impaired cardiac function. Patients have been successfully resuscitated in this situation with large doses of adrenaline and noradrenaline, ${ }^{8}$ amrinone, ${ }^{18}$ glucagon, ${ }^{19}$ and mechanical (intra-aortic balloon pump) support. ${ }^{20}$ Our findings of pronounced ECG changes in a reaction without cardiovascular compromise or subsequent evidence of coronary disease is consistent with the increasing recognition of the human heart, which contains significant numbers of mast cells, ${ }^{21}$ as a major shock organ in some cases of anaphylaxis. ${ }^{22} 23$ 

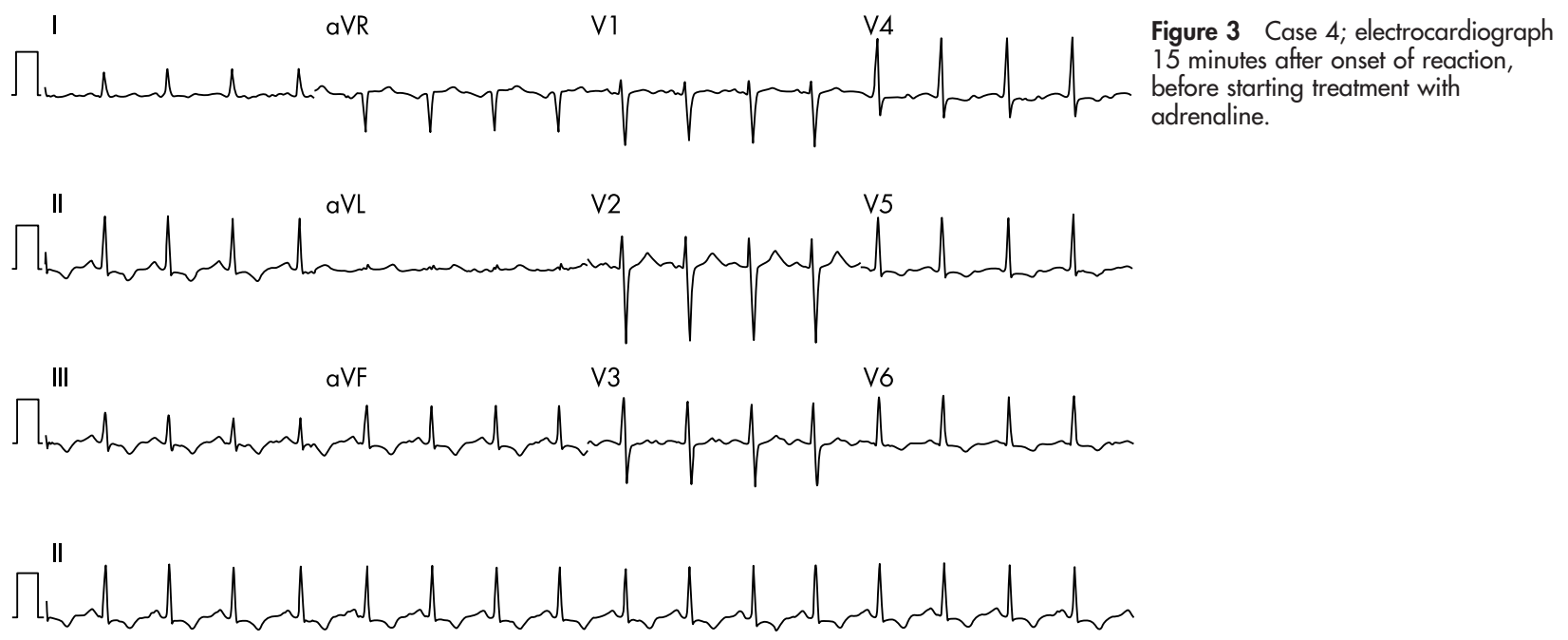

Relative bradycardia (falling heart rate despite worsening hypotension) has been reported previously in the setting of deliberately induced sting anaphylaxis, ${ }^{4}{ }^{8}$ but may be underrecognised clinically where a rapid demise occurs before reaching medical care. For example, a recent sting death observed by us was characterised by sudden collapse with a severe bradycardia manifested as a slow idioventricular rhythm noted five minutes later on the arrival of paramedics; at the time we attributed this finding to hypoxia. ${ }^{15}$

Possible explanations for the bradycardia identified here include an effect of anaphylactic mediators on the heart or nervous system, or both, and neurocardiogenic mechanisms. Bradycardia may be a non-specific feature of severe hypovolaemic-distributive shock. Physiological studies of awake mammals have identified two phases of response to hypovolaemia, an initial phase of blood pressure maintenance by tachycardia and peripheral vasoconstriction, followed by a second phase with more severe hypovolaemia that is characterised by bradycardia, reduced peripheral vascular tone and a profound fall in blood pressure. ${ }^{24}$

The mechanisms involved may be similar to those implicated in neurocardiogenic syncope. In that condition, excessive activation of the cardiac mechanoreceptors by mechanical stimulation (increased force of contraction) and chemical factors during a period of sympathetic excitation is

\section{What this paper adds}

- Although adrenaline and volume resuscitation are considered a cornerstone of treatment for anaphylaxis, no prospective analysis of such an approach has previously been performed. The pathophysiology of anaphylaxis in humans has also been difficult to study.

- This prospective study demonstrates that a carefully titrated adrenaline infusion combined with volume resuscitation is effective treatment for anaphylaxis. It also lays the foundation for further research into the use of corticosteroids and antihistamines for terminating the underlying reaction, and perhaps a comparison between intramuscular and intravenous adrenaline. Observations during this study implicate a neurocardiogenic mechanism for some lethal reactions. Intravenous atropine may have a supplementary role when hypotension is combined with bradycardia. thought to combine with potentiated central reflexes to trigger both a parasympathetic outflow and a dramatic reduction in sympathetic nerve outflow. ${ }^{25}$ Catecholamines and prostaglandins appear to sensitise cardiac mechanoreceptors, while serotonin (5-hydroxytryptamine) and nitric oxide have been found to potentiate the central reflexes. ${ }^{26}$ Levels of these mediators are known to be elevated during anaphylaxis. ${ }^{4728}$ Thus, during anaphylaxis neurocardiogenic mechanisms may both exacerbate peripheral vasodilatation and cause bradycardia. This may be lethal when combined with other features of anaphylactic shock. Bradycardia has not been reported as a major feature of anaphylaxis under anaesthesia, ${ }^{7}$ perhaps related to the blunting of central reflexes in that setting, or because such physiological changes are less likely to be identified in retrospective studies.

Extrapolation of our findings to other forms of anaphylaxis and to patients with comorbidities should be done with caution. There is some evidence that anaphylaxis to ingested antigens is more likely to involve severe bronchospasm, ${ }^{6}$ and delayed phase reactions, ${ }^{29}$ where additional bronchodilator treatment, corticosteroids, and prolonged periods of observation may be required. Practitioners should also be aware of the phenomenon of adrenaline resistant anaphylaxis and consider additional treatment measures including more aggressive volume resuscitation, higher doses of adrenaline, noradrenaline, glucagon, amrinone, and balloon pump support. Furthermore, our study was performed under the supervision of emergency medicine specialists in a well equipped resuscitation room. Our treatment protocol may not be applicable to other clinical settings.

Our research supports the use of anaphylaxis treatment protocols that incorporate oxygen, intravenous adrenaline infusions and volume resuscitation. We also found evidence of inappropriately high parasympathetic (vagal) tone, suggesting a supplementary role for atropine in cases associated with severe bradycardia. Our treatment model could be used to investigate the use of corticosteroids and antihistamines to terminate the underlying reaction, using the recurrence of symptoms when first ceasing the adrenaline infusion as an end point. A direct comparison of intravenous versus intramuscular adrenaline may also be warranted.

\section{ACKNOWLEDGEMENTS}

We thank cardiologist A/Prof Philip Roberts-Thomson for his assistance managing our patients with ECG changes, and our pharmacist Michael Wiese, research assistant Sandra Ahokas and medical honours student Andrew Black for their assistance with immunotherapy and sting challenges. We also thank Professor Malcolm Fisher for reviewing the manuscript. 


\section{Contributors}

$\mathrm{S}$ Brown was responsible for study design and performed sting challenges and data analysis. K Blackman and V Stenlake assisted with protocol design and performed sting challenges. R Heddle assisted with study design and provided clinical support during the trial. All investigators contributed to writing of the report.

\section{Authors' affiliations}

S G A Brown, K E Blackman, V Stenlake, Department of Emergency Medicine, Royal Hobart Hospital, Hobart, Australia

R J Heddle, Department of Immunology, Allergy and Arthritis, Flinders Medical Centre and Flinders University, Bedford Park, Australia

Funding: this research was supported by the Royal Hobart Research Foundation, the Dick Butfield Memorial Scholarship (awarded to S Brown), NSL Health Limited (Melbourne) and a donation by Cosy Cabins Tasmania in the memory of Mr Arthur F Park who died following a jack jumper sting in 1999.

Conflicts of interest: none declared.

\section{REFERENCES}

1 Project team of the Resuscitation Council (UK). Emergency medical treatment of anaphylactic reactions. Resuscitation 1999:41:93-9.

2 The American Heart Association in collaboration with the International Liaison Committee on Resuscitation. Guidelines 2000 for cardiopulmonary resuscitation and emergency cardiovascular care. Part 8: advanced challenges in resuscitation: section 3: special challenges in ECC. Anaphylaxis. Circulation 2000;102(suppl 8):1241-3.

3 Project team of the Resuscitation Council (UK). Update on the emergency medical treatment of anaphylactic reactions for first medical responders and for community nurses. Emerg Med J 2001;18:393-5.

4 van der Linden PW, Struyvenberg A, Kraaijenhagen RJ, et al. Anaphylactic shock after insect-sting challenge in 138 persons with a previous insect-sting reaction. Ann Intern Med 1993;1 18:161-8.

5 Sampson HA, Mendelson L, Rosen JP. Fatal and near-fatal anaphylactic reactions to food in children and adolescents. N Engl J Med 1992;327:380-4.

6 Pumphrey RS. Lessons for management of anaphylaxis from a study of fatal reactions. Clin Exp Allergy 2000;30:1 144-50.

7 Fisher MM. Clinical observations on the pathophysiology and treatment of anaphylactic cardiovascular collapse. Anaesth Intensive Care 1986;14:17-21.

8 Smith PL, Kagey-Sobotka A, Bleecker ER, et al. Physiologic manifestations of human anaphylaxis. J Clin Invest 1980;66:1072-80.
9 Bautista E, Simons FE, Simons KJ, et al. Epinephrine fails to hasten hemodynamic recovery in fully developed canine anaphylactic shock. Int Arch Allergy Immunol 2002;128:151-64.

10 Brown AF. Anaphylaxis: quintessence, quarrels, and quandaries. Emerg Med J 2001; 18:328

11 Fisher $M$. Treating anaphylaxis with sympathomimetic drugs. BMJ 1992;305: 1107-8.

12 Fisher $\mathbf{M}$. Treatment of acute anaphylaxis. Letters contained errors of logic. BMJ 1996;312:637-8.

13 Brown SGA, Wiese MD, Blackman KE, et al. Ant venom immunotherapy: a double-blind, placebo-controlled, crossover trial. Lancet 2003;361:1001-6.

14 Muller U. Insect sting allergy: clinical picture, diagnosis and treatment. New York: Gustav Fischer Verlag, 1990.

15 Brown SGA, Wu QX, Kelsall GR, et al. Fatal anaphylaxis following jack jumper ant sting in southern Tasmania. Med J Aust 2001;175:644-7.

16 van der Linden PW, Hack CE, Struyvenberg A, et al. Insect-sting challenge in 324 subjects with a previous anaphylactic reaction: current criteria for insectvenom hypersensitivity do not predict the occurrence and the severity of anaphylaxis. J Allergy Clin Immunol 1994;94:151-9.

17 Blaauw PJ, Smithuis OL, Elbers AR. The value of an in-hospital insect sting challenge as a criterion for application or omission of venom immunotherapy. J Allergy Clin Immunol 1996;98:39-47.

18 Otero E, Onufer JR, Reiss CK, et al. Anaphylaxis-induced myocardial depression treated with amrinone. Lancet 1991;337:682.

19 Zaloga GP, Delacey W, Holmboe E, et al. Glucagon reversal of hypotension in a case of anaphylactoid shock. Ann Intern Med 1986;105:65-6.

20 Raper RF, Fisher MM. Profound reversible myocardial depression after anaphylaxis. Lancet 1988;i:386-8.

21 Patella V, Marino I, Lamparter B, et al. Human heart mast cells. Isolation, purification, ultrastructure, and immunologic characterization. J Immunol 1995; 154:2855-65.

22 Cooper DJ. Cardiac dysfunction during anaphylaxis in patients. Appl Cardiopulm Pathophysiol 1993;5:9-18.

23 Kemp SF, Lockey RF. Anaphylaxis: a review of causes and mechanisms. J Allergy Clin Immunol 2002;1 10:341-8.

24 Schadt JC, Ludbrook J. Hemodynamic and neurohumoral responses to acute hypovolemia in conscious mammals. Am J Physiol 1991;260:H305-18.

25 Abboud FM. Ventricular syncope: is the heart a sensory organ? N Engl J Med 1989;320:390-2.

26 Abboud FM. Neurocardiogenic syncope. N Engl J Med 1993;328:1117-20.

27 Mitsuhata H, Shimizu R, Yokoyama MM. Role of nitric oxide in anaphylactic shock. J Clin Immunol 1995;15:277-83.

28 van der Linden PW, Hack CE, Poortman J, et al. Insect-sting challenge in 138 patients: relation between clinical severity of anaphylaxis and mast cell activation. J Allergy Clin Immunol 1992;90:110-18.

29 Stark BJ, Sullivan TJ. Biphasic and protracted anaphylaxis. J Allergy Clin Immunol 1986;78:76-83. 